\title{
AN ELEMENTARY PROOF ABOUT THE ORDER OF THE ELEMENTS IN A DISCRETE GROUP
}

\author{
G. CROMBEZ
}

\begin{abstract}
We give an elementary direct proof of the following property: if for a discrete group $G$ some $l_{p}(G)$-space $(1<p<\infty)$ is an algebra, then all elements of $G$ have uniformly bounded order.
\end{abstract}

If $G$ is a discrete group and $l_{p}(G)(1<p<\infty)$ is an algebra under convolution, then the property that all elements of $G$ have uniformly bounded order is usually proved in an indirect way, by first showing that $G$ is a Burnside group [1] (i.e., for any Haar measure $\mu$ on $G$ there exists a constant $C_{\mu}>0$ such that $\mu(A B) \geqslant$ $C_{\mu} \mu(A) \mu(B)$ for all compact subsets $A, B$ of $G$ ), and then using the special properties of a Burnside group. We give here an elementary direct proof of the mentioned property. We make use of the well-known fact that, if for a locally compact group $G$ and some $p(1<p<\infty) L_{p}(G)$ is an algebra under convolution, there exists a constant $C>0$ such that $\|f * g\|_{p} \leqslant C\|f\|_{p}\|g\|_{p}\left(f, g \in L_{p}(G)\right)$.

LemMa. For $1<p<\infty$ we have

$$
\lim _{n \rightarrow \infty} \frac{1^{p}+2^{p}+\cdots+(n-1)^{p}+n^{p}+(n-1)^{p}+\cdots+1^{p}}{n^{2}}=\infty .
$$

Proof. The result is certainly true for $p=2$ (since then the numerator is of order $n^{3}$ ), and so also for $p>2$; on the contrary, it is not true for $p=1$. In order to prove it for $1<p<2$ it is sufficient to prove the inequality

$$
1^{p}+2^{p}+\cdots+(n-1)^{p}+n^{p}+(n-1)^{p}+\cdots+1^{p}>\frac{n^{p}(n-2)}{2},
$$

for sufficiently great values of $n(1<p<2)$, and (1) will be true as soon as

$$
1^{p}+2^{p}+\cdots+(n-1)^{p}>\frac{n^{p}(n-4)}{4}, \text { for } n \geqslant 5 .
$$

Now the truth of (2) may be shown by induction. For (2) is certainly true for $n=5$. So, assuming that

$$
1^{p}+2^{p}+\cdots+(n-2)^{p}>\frac{(n-1)^{p}(n-1-4)}{4},
$$

we have

$$
1^{p}+2^{p}+\cdots+(n-2)^{p}+(n-1)^{p}>\frac{(n-1)^{p}(n-1)}{4},
$$

Received by the editors March 17, 1981.

1980 Mathematics Subject Classification. Primary 43A15: Secondary 22D05.

Kel words and phrases. Convolution, discrete group, order of an element.

(c) 1982 American Mathematical Society 0002-9939/81/0000-1115/\$01.50 
and hence (2) will be true as soon as $(n-1)^{p}(n-1)>n^{p}(n-4)$, or

$$
(1-1 / n)^{p}>1-3 /(n-1) \text {. }
$$

But (3) is valid since $(1-1 / n)^{p}>(1-1 / n)^{2}$ for $1<p<2$, and since it is obvious that $(1-1 / n)^{2}>1-3 /(\mathrm{n}-1)$.

Let then $G$ be a discrete group with identity $e$ such that $l_{p}(G)$ is an algebra $(1<p<\infty)$, and suppose that the elements of $G$ do not have uniformly bounded order. Then, for each $n \in Z^{+}$there exists a $y \in G$ such that $e \notin\left\{y, y^{2}, \ldots, y^{2 n}\right\}$. Put $f=\sum_{i=1}^{n} \delta_{y} i$, where $\delta_{y} i(x)=1$ for $x=y^{i}$ and zero in the other points of $G$. Then $\|f\|_{p}=n^{1 / p}$, while a calculation shows that $(f * f)\left(y^{2}\right)=1, \quad(f * f)\left(y^{3}\right)=$ $2, \ldots,(f * f)\left(y^{n+1}\right)=n, \ldots,(f * f)\left(y^{2 n}\right)=1$, and $(f * f)(x)=0$ when $x \notin$ $\left\{y^{2}, y^{3}, \ldots, y^{2 n}\right\}$. Hence $\|f * f\|_{p}=\left(1^{p}+\cdots+n^{p}+\cdots+1^{p}\right)^{1 / p}$, and so

$$
\frac{\|f * f\|_{p}}{\|f\|_{p}\|f\|_{p}}=\left(\frac{1^{p}+2^{p}+\cdots+n^{p}+(n-1)^{p}+\cdots+1^{p}}{n^{2}}\right)^{1 / p} \rightarrow \infty \text { for } n \rightarrow \infty,
$$

a contradiction.

ACKNOWLEDGEMENT. The author expresses his appreciation to the referee for a valuable suggestion.

\section{REFERENCES}

1. W. Żelasko, On the Burnside problem for locally compact groups. Symposia Mathematica 16. Academic Press, New York, 1975, pp. 409-416.

Seminar of Higher Analysis, State University of Ghent, Galglaan 2, B-9000 Ghent, Belgium 